\title{
LncRNA RHPN1-AS1 promotes cholangiocarcinoma progression and predicts poor clinical outcome through miR-345-5p/YAP1 axis
}

\section{Lifeng Ma}

the Second Hospital of Hebei Medical University

Tao Li

the Second Hospital of Hebei Medical University

Guochao Liu

the Second Hospital of Hebei Medical University

Jianlong Wang

the Second Hospital of Hebei Medical University

Zhaoqiang Yin

the Second Hospital of Hebei Medical University

Jiansheng Kang ( $\sim$ kangjiansheng62@163.com )

the Second Hospital of Hebei Medical University https://orcid.org/0000-0002-9694-4481

Research

Keywords: cholangiocarcinoma (CCA), RHPN1-AS1, miR-345-5p, prognosis, pathway

Posted Date: August 20th, 2020

DOI: https://doi.org/10.21203/rs.3.rs-58822/v1

License: (c) (i) This work is licensed under a Creative Commons Attribution 4.0 International License.

Read Full License 


\section{Abstract \\ Background}

LncRNAs have proven to be involved in the initiation and progression of cholangiocarcinoma (CCA), although the mechanism by which this occurs remains unknown.

\section{Methods}

The current study reveals that RHPN1-AS1 was overexpressed in CCA patient samples, which predicted poor outcome of CCA patients. RHPN1-AS1 increased in vitro pancreatic carcinoma cell proliferation as well as promoted xenograft growth in vivo. Mechanistically, DANCR upregulated expression of YAP1 by competitively binding to miR-345-5p. Importantly, RHPN1-AS1 level was positively correlated with YAP1 expression level in CCA tissues. Moreover, YAP1 overexpression could predicted a poor outcome of CCA patients.

\section{Results}

Taken together, our results suggested that RHPN1-AS1 might be a remarkable biomarker to evaluate prognosis in CCA.

\section{Conclusion}

The RHPN1-AS1/YAP1 axis may provide new strategies for CCA clinical practice.

\section{Background}

Cholangiocarcinoma (CCA) is a devastating biliary malignancy with an abysmal overall 5-year survival rate at stages III and IV of less than 10\%, [1]. CCA is one of the most difficult intra-abdominal cancers, occurring via the malignant transformation of the epithelium lining of the biliary tree, called cholangiocytes, that derive from the bile ductules [2, 3]. Histologically, CCAs can be classified into intrahepatic cholangiocarcinoma (iCCA), distal cholangiocarcinoma (dCCA) and perihilar cholangiocarcinoma (pCCA) subtypes [4]. Despite the advancements and progress in the treatment strategies, including neoadjuvant chemotherapy and radical surgery, the clinical outcome remains poor [2]. In addition, the important role of non-coding RNA dysregulation in the development of CCA has also received increasing attention.

Long non-coding RNAs (LncRNAs), more than 200 nucleotides in length are key regulators in a wide range of biological activities and cancer progression [5]. Recent studies have found that IncRNAs are showing abnormal abundances in samples of different types of tumors, which are emerging as promising 
therapeutic targets in modulating in tumor proliferation, cell migration, metastasis, and apoptosis [6, 7]. Although multiple IncRNA networks have been shown to participate in the metastasis, proliferation, and apoptosis is of CCA cells. However, possible IncRNA networks in CCA and their related mechanisms are still unclear.

RHPN1-AS1 has a length of 2030 bp transcript located in human chromosome 8q24.3, which is an antisense IncRNA originated from the promoter region of RHPN1 [8]. Nevertheless, the mechanistic role of RHPN1-AS1 in CCA has not yet been elucidated. In this study, we confirmed the upregulation of RHPN1AS1 in CCA and explored the hypothesis that RHPN1-AS1 participates in CCA progression as a candidate epigenetic diagnostic biomarker.

\section{Materials And Methods}

\section{Tissue sample}

Frozen fresh paraffin-embedded CCA and para-cancerous tissues of 34 CCA patients were collected during January 2013 to October 2015 at the Second Hospital of Hebei Medical University. This research has been carried out in accordance with the World Medical Association Declaration of Helsinki. Informed written consent was obtained from each subject. This study was approval by the medical ethics committee of the Second Hospital of Hebei Medical University.

\section{Cell culture and transfection}

CCA cell lines, including RBE, TFK-1, HCCC9810, QBC939, HUCCT1 and normal human bile duct cell line (HiBEC), as well as HEK293T cells were all purchased from ATCC (Rockville, Maryland, USA). The cells were cultured in RPMI-1640 (Gibco, Carlsbad, CA, USA) supplemented with 10\% fetal bovine serum.

\section{Establishment of Xenograft model in nude mice}

Animal research has been approved by the Second Hospital of Hebei Medical University Animal Care and Use Committee (IACUC). To establish a xenograft tumor model, 5 mice were divided into each group. 200 $\mu \mathrm{l}$ of $1 \times 106$ cells were injected subcutaneously into the axilla of female Balb / C nude mice. After six weeks, the mice were sacrificed by $\mathrm{CO} 2$ asphyxiation and the subcutaneous tumor weight was measured.

\section{qRT-PCR method}

Total RNA was extracted with triazole reagent (Invitrogen, Carlsbad, CA, USA). CDNA was generated using PrimeScriptRT kit (Thermo Scientific, Glen Brunie, MA, USA). qRT-PCR was performed using the TB Green real-time PCR kit (RR420A, Takara) according to the manufacturer's procedures. GAPDH serves as an internal reference for cytoplasmic gene expression. This expression is calculated by the $2-\Delta \mathrm{Ct}$ method.

\section{Cell proliferation assays}


QBC939 and HUCCT1 cells were seeded at a density of $1 \times 105$ cells/well into 96-well Plates (Corning, New York, USA). Cell proliferation was analyzed using the cell counting kit-8 (CCK-8, Guangzhou, China) according to the manufacturer's instructions.

\section{Tumor sphere assay}

QBC939 and HUCCT1 cells were plated into 24-well plates (Corning, New York, USA) as a single-cell suspension and contained 2\% B27 (Life Technology), 20ng / mL epidermal growth factor (Life Technology) and 20ng / mL bFGF was added into Dulbecco's modified Eagle's medium / F-12.

\section{Western Blot}

Proteins were extracted as previously described. Equal amounts of protein are was subjected to electrophoresis and subsequently transferred to a polyvinylidene fluoride membrane (Schwabacher Merck Micropore, Germany). Antibodies to CDK8, MST1, YAP1and TEAD1, p-YAP came from cell signaling technology. The antibody to $\beta$-actin was from Sigma. HRP-conjugated secondary antibodies are from Thermo.

\section{Luciferase assay}

PMir-RHPN1-AS1-WT or PMir-RHPN1-AS1-Mut were co-transfected into HEK293T cells by pmirGLO-Report luciferase vector. Transfection of miR-345-5p-mimics were performed using Lipofectamine 3000 according to the manufacturer's instructions. After transfection for $48 \mathrm{hrs}$, the Renilla and Firefly luciferase activities could be determined through the Dual-Luciferase Reporter Assay System according to the manufacturer's protocol.

\section{In situ hybridization (ISH)}

In situ hybridization was applied to examine the expression of RHPN1-AS1 as well as miR-345-5p on tissue sections according to the manufacturer's procedures (BOSTER, Hubei, China) as previously described [9].

\section{Immunochemistry (IHC) assay}

The tumor sections were dried and dewaxed and were washed with $3 \%$ hydrogen peroxide (Sigma) diluted in methanol, and then incubated and heated in sodium phosphate buffer ( $\mathrm{pH}$ 6.0). Samples were incubated with LATS1 (1:200, CST, Danvers, USA) primary antibodies overnight at $4{ }^{\circ} \mathrm{C}$, and then performed using a non-biotin horseradish peroxidase detection system according to the manufacturer's instructions.

\section{Statistical Analysis}

The data are presented as mean $\pm S D$, and we calculated the comparison by Student's $t$ test and $x 2$ test for comparison of the differences between groups. The Kaplan-Meier method was applied to plot the 
survival curves. Statistical analysis was performed using GraphPad Prism 5.0 (GraphPad Software, LaJolla, CA, USA). P $<0.05$ was recognized as having statistically significant difference.

\section{Results}

\section{RHPN1-AS1 as a IncRNA was upregulated in CCA}

First, RHPN1-AS1 levels were measured among 34 paired CCA specimen and matching non-cancer tissue samples. Compared with non-tumor tissues, RHPN1-AS1 levels in primary CCA tissues were significantly enhanced (Fig.1A). Consistently, compared to that in normal HiBEC cells, the expression of RHPN1-AS1 in TFK-1, RBE, QBC939, HCCC9810 and HUCCT1 cell lines were significantly higher (Fig.1B). Analysis shows that RHPN1-AS1 consists of an exon (Ensemble: ENSG0000254389), located in the cytoplasm and nucleus (Fig.1C).

\section{RHPN1-AS1 promoted proliferation of CCA cell lines}

Next, gain- and loss-of function studies were implemented to determine the functional role of RHPN1-AS1 in CCA cells, Firstly, we silenced the expression of RHPN1-AS1 by transfection with sh-1\# or sh-2\# shRNAs in HUCCT1 and QBC939 cell lines (Fig.2A). MTS assay showed that the proliferation of CCA cells was decreased after RHPN1-AS1 knockdown (Fig.2B). Secondly, colony formation assay demonstrated that RHPN1-AS1 knockdown greatly decreased colony formation ability of CCA cells (Fig.2C). Moreover, RHPN1-AS1 overexpression remarkedly enhanced cell proliferative rates as well as colony formation capacity in TFK-1 cells (Fig.2D). Furthermore, the tumor sphere numbers and diameters of CCA cells were greatly decreased after RHPN1-AS1 knockdown, whereas remarkably increased after RHPN1-AS1 overexpression, showing that RHPN1-AS1 inhibited tumor sphere formation of CCA cells (Fig.2E-F). These data together indicated that RHPN1-AS1 had driven the proliferation of CCA cells.

\section{RHPN1-AS1 enables CCA cells proliferation via activating YAP1}

To elucidate the regulatory role of RHPN1-AS1, we determined the downstream key protein expression levels. As shown in Fig.3A, RHPN1-AS1 knockdown negatively regulated the protein level of YAP1 without showing any influence on the protein levels of YAP1 upstream kinases CDK8 and MST1. The mRNA level of YAP1 was greatly lower in RHPN1-AS1 knockdown group compared with the control group (Fig.3B). Immunofluorescence staining result showed that YAP1 expression and nuclear translocation were greatly repressed after RHPN1-AS1 knockdown (Fig.3C). Conversely, ectopic overexpression of RHPN1-AS1 positively regulated YAP1 expression at both protein and mRNA levels (Fig.3D), but negatively regulated YAP1 serine 127 phosphorylation. Furthermore, Immunofluorescence staining revealed that the YAP1 protein level as well as the nuclear translocation of RHPN1-AS1 were significantly enhanced after RHPN1AS1 overexpression (Fig.3E). Consistently, PCR results showed the YAP1 mRNA expression level was also higher in the primary CCA tissues (Fig.3F). Moreover, we found that YAP1 overexpression can reverse the impacts of RHPN1-AS1 knockdown on CCA cell proliferation and tumor sphere formation (Fig.3G-H). 
However, YAP1 knockdown showed the opposite effects (Fig.3I-J). Those data suggested that RHPN1AS1 had a role in CCA cells through activating YAP1 activity.

\section{RHPN1-AS1 promoted YAP1 expression via sponging miR-345-5p}

To detect whether RHPN1-AS1 can function as a miRNA sponge to upregulate the YAP1 expression level, the bioinformatic analysis LncBase Predicted v. 2 was implemented and miR-345-5p was predicted to target RHPN1-AS1 (Fig.4A). Subsequently, RIP assay proved that miR-345-5p was associated with RHPN1-AS1 via targeting binding of $3^{\prime}$-UTR (Fig.4B). RIP assays also uncovered that miR-345-5p could endogenously bind to YAP1 3'-UTR specifically (Fig.4C). Furthermore, we found that the luciferase activity of HEK-293T cell in RHPN1-AS1 WT group was significantly lower after transfected with miR-345-5p mimics (Fig.4D). To evaluate whether YAP1 can be a direct target of miR-345-5p, reporters containing WT/Mut YAP1 3'-UTR mRNA were co-transfected with miR-345-5p mimics into HEK-293T cells. Our data showed that the reporter activity was deceased in pMir-YAP1-WT group co-transfected with miR-345-5p mimics (Fig.4E), suggesting that YAP1 3'-UTR mRNA was inhibited by miR-345-5p. Western bolt showed that miR-345-5p mimics strongly decreased the protein levels of YAP1 in QBC939 and HUCCT1 cells, whereas miR-345-5p inhibitor presented the opposite effect (Fig.4F-G).

\section{YAP1/TEAD1 complex feedback transactivates RHPN1-AS1}

Next, we wondered if YAP1/TEAD1 could reversely influence the level of RHPN1-AS1 expression. As expected, YAP1 knockdown downregulated RHPN1-AS1 levels but was upregulated after YAP1 overexpression in HUCCT1 and QBC939 cells (Fig.5A), suggesting that YAP1 could have positive regulatory impact on RHPN1-AS1 transcription. Subsequently, TEAD1 knockdown reduced YAP1 enrichment in the promoter region of RHPN1-AS1 (Fig.5B). Moreover, TEAD1 knockdown abolished the upregulation of RHPN1-AS1 expression by YAP1 overexpression (Fig.5C-D). These data revealed that YAP1/TEAD1 complex feedback transactivates RHPN1-AS1 in CCA cells.

\section{RHPN1-AS1 promoted CCA progression through YAP1 and correlated with poor outcome in CCA patients}

Next, sh-RHPN1-AS1 and sh-Con transfected QBC939 cells were subcutaneously injected into nude mice to see the role of RHPN1-AS1 in vivo. Fig.6A showed that RHPN1-AS1 knockdown repressed tumor growth, however rescue assay showed that tumor growth in sh-2\#+YAP1 group was greatly increased compared to sh-2\#+vector group. Moreover, RHPN1-AS1 and miR-345-5p expression levels were examined by ISH in CCA patients. About 85\% tissues were RHPN1-AS1 expression positive among all the specimens. RHPN1-AS1 was detected in both cytoplasm and nuclear (Fig.6B). ISH showed that there was a positive correlation between RHPN1-AS1 and YAP1 expressions (Fig.6C). Notably, both high RHPN1AS1 as well as YAP1 protein level predicted a poor outcome of CCA patients (Fig.6D). This result suggested that RHPN1-AS1/YAP1 axis was highly correlated with CCA tumor growth and poor outcome of CCA patients.

\section{Discussion}


The current research illustrated the key fundamental role of IncRNA-RHPN1-AS1 in proliferation and progression of CCA. Our findings for the first time shed some deep lights on the underlying mechanism of CCA: RHPN1-AS1 was overexpressed in CCA and promoted the growth of CCA by enhancing YAP1 activity; RHPN1-AS1 as a competitive endogenous RNA (ceRNA) up-regulated the expression of YAP1 ; RHPN1-AS1 interacted with YAP1, blocks CDK8-mediated YAP1 inactivation, YAP1 / TEAD1 complex transcription regulated RHPN1-AS1 expression, which formed a feedback loop among CCA.

LncRNAs play some vital roles in cancer, especially malignant transformation, invasiveness, and epithelial-mesenchymal transition [10-12]. According to reports, some IncRNAs could regulate tumorigenesis, including apoptosis, metastasis, proliferation as well as drug resistance [13]. Emerging studies showed that LncRNAs are widely reported as ceRNAs to sponge miRNAs, which in turn modulate the expression of target genes $[14,15]$. LncRNA DLGAP1-AS1 regulated by JAK2 / STAT3 triggered the invasive and metastatic cascades of liver cancer by competitively binding miR-26a and b-5p / IL-6, thereby inducing the EMT process [16]. GAS5 inhibited the progress of colorectal cancer by promoting the YAP activation by $\mathrm{m}^{6} \mathrm{~A}$ modification. HNF1A-AS1 acted as a sponge for miR-34b and induced TUFT1 expression to mediate cervical cancer cisplatin resistance [17]. RHPN1-AS1 has been shown to play oncogenic role in various cancers [18-20].

In our study, RHPN1-AS1 shared a miR-345-5p response element with YAP1. Experiments show that YAP1 is the real target of miR-345-5p, miR-345 The functional inhibition of -5p effectively rescued the expression of YAP1 induced by RHPN1-AS1 and reduced after RHPN1-AS1 knockout in CCA cells, suggesting that RHPN1-AS1 acted as a ceRNA. We also verified that RHPN1-AS1 knockdown increased YAP1 phosphorylation, which was the key downstream component of the Hippo signaling pathway. YAP1 is also reported as oncogene in different tumors and could be post-transcriptionally regulated by cytoplasmic sequestration or kinases mediated degradation. To verify the function of RHPN1-AS1 on YAP1 protein, miR-345-5p inhibitor was used to examine its impact on YAP1 expression. The experimental results indicated that RHPN1-AS1 could negatively regulated YAP1 phosphorylation in CCA cells. Furthermore, we identified RHPN1-AS1 targeted bind to YAP1 protein, which inhibited the phosphorylation activated by CDK8. This indicated that RHPN1-AS1 regulated YAP1 signaling either by enhancing expression or modulating function in CCA. Finally, mechanistic study showed that the suppressive effect of RHPN1-AS1 knockdown on YAP1 expression was partially restored with miR-345-5p inhibition (Fig. 7).

\section{Conclusion}

IncRNA RHPN1-AS1 was aberrantly upregulated in CCA and predicted poor clinical outcome. RHPN1-AS1 improved CCA progression via modulating YAP1 signaling. RHPN1-AS1 promoted YAP1 activity through sponging miR-345-5p as well as by binding YAP1 therefore elevating activation. This study indicated RHPN1-AS1/YAP1 axis was a promising treatment target of CCA.

\section{Abbreviations}


cholangiocarcinoma (CCA); intrahepatic cholangiocarcinoma (iCCA); distal cholangiocarcinoma (dCCA); perihilar cholangiocarcinoma (pCCA); Long non-coding RNAs (LncRNAs); human bile duct cell line (HiBEC); In situ hybridization (ISH)

\section{Declarations}

\section{Ethics approval and consent to participate}

This research has been carried out in accordance with the World Medical Association Declaration of Helsinki and that all subjects provided written informed consent. This study was approval by the medical ethics committee of the Second Hospital of Hebei Medical University.

\section{Consent for publication}

Informed consent was obtained from all individual participants included in the study.

\section{Availability of data and material}

The authors declare that the data supporting the findings of this study are available within the article.

\section{Competing interests}

The authors declare that they have no competing interests.

\section{Funding}

None.

\section{Authors' contributions}

MLF carried out the molecular studies and drafted the manuscript. LT and YZQ carried out the animal studies. LGC and WJL participated in performing the statistical analysis. KJS and MLF YZQ conceived of the study and participated in its design and coordination. All authors read and approved the final manuscript.

\section{Acknowledgements}

We thank Prof. Cheng Bing for statistical support and tissue pathological verification.

\section{References}


1. Altman AM, Kizy S, Marmor S, Huang JL, Denbo JW, Jensen EH. Current survival and treatment trends for surgically resected intrahepatic cholangiocarcinoma in the United States. J Gastrointest Oncol. 2018;9:942-52.

2. Wakizaka K, Yokoo H, Kamiyama T, Ohira M, Kato K, Fujii Y, Sugiyama K, Okada N, Ohata T, Nagatsu A, Shimada S, Orimo T, Kamachi $\mathrm{H}$, et al. Clinical and pathological features of combined hepatocellular-cholangiocarcinoma compared with other liver cancers. J Gastroenterol Hepatol. 2019;34:1074-80.

3. Yoh T, Hatano E, Kasai Y, Fuji H, Nishi K, Toriguchi K, Sueoka H, Ohno M, Seo S, Iwaisako K, Taura K, Yamaguchi R, Kurokawa M, et al. Serum Nardilysin, a Surrogate Marker for Epithelial-Mesenchymal Transition, Predicts Prognosis of Intrahepatic Cholangiocarcinoma after Surgical Resection. Clin Cancer Res. 2019;25:619-28.

4. Chang WW, Hsiao PK, Qin L, Chang CL, Chow JM, Wu SY. Treatment outcomes for unresectable intrahepatic cholangiocarcinoma: Nationwide, population-based, cohort study based on propensity score matching with the Mahalanobis metric. Radiother Oncol. 2018;129:284-92.

5. Peng WX, Koirala P, Mo YY. LncRNA-mediated regulation of cell signaling in cancer. Oncogene. 2017;36:5661-7.

6. Bhan A, Soleimani M, Mandal SS. Long Noncoding RNA and Cancer: A New Paradigm. Cancer Res. 2017;77:3965-81.

7. Sanchez Calle A, Kawamura Y, Yamamoto Y, Takeshita F, Ochiya T. Emerging roles of long noncoding RNA in cancer. Cancer Sci. 2018;109:2093-100.

8. Qiu X, Lei Z, Wang Z, Xu Y, Liu C, Li P, Wu H, Gong Z. Knockdown of LncRNA RHPN1-AS1 Inhibits Cell Migration, Invasion and Proliferation in Head and Neck Squamous Cell Carcinoma. J Cancer. 2019;10:4000-8.

9. Geng H, Bu HF, Liu F, Wu L, Pfeifer K, Chou PM, Wang X, Sun J, Lu L, Pandey A, Bartolomei MS, De Plaen IG, Wang P, et al. In Inflamed Intestinal Tissues and Epithelial Cells, Interleukin 22 Signaling Increases Expression of H19 Long Noncoding RNA, Which Promotes Mucosal Regeneration. Gastroenterology. 2018;155:144-55.

10. Liang L, Xu J, Wang M, Xu G, Zhang N, Wang G, Zhao Y. LncRNA HCP5 promotes follicular thyroid carcinoma progression via miRNAs sponge. Cell Death Dis. 2018;9:372.

11. Robertson AG, Kim J, Al-Ahmadie H, Bellmunt J, Guo G, Cherniack AD, Hinoue T, Laird PW, Hoadley KA, Akbani R, Castro MAA, Gibb EA, Kanchi RS, et al. Comprehensive Molecular Characterization of Muscle-Invasive Bladder Cancer. Cell. 2017; 171: 540 - 56 e25.

12. Li J, Li Z, Zheng W, Li X, Wang Z, Cui Y, Jiang X. LncRNA-ATB: An indispensable cancer-related long noncoding RNA. Cell Prolif. 2017; 50.

13. Liang H, Yu T, Han Y, Jiang H, Wang C, You T, Zhao X, Shan H, Yang R, Yang L, Shan H, Gu Y. LncRNA PTAR promotes EMT and invasion-metastasis in serous ovarian cancer by competitively binding miR-101-3p to regulate ZEB1 expression. Mol Cancer. 2018;17:119. 
14. Song W, Gu Y, Lu S, Wu H, Cheng Z, Hu J, Qian Y, Zheng Y, Fan H. LncRNA TRERNA1 facilitates hepatocellular carcinoma metastasis by dimethylating $\mathrm{H} 3 \mathrm{~K} 9$ in the $\mathrm{CDH} 1$ promoter region via the recruitment of the EHMT2/SNAI1 complex. Cell Prolif. 2019;52:e12621.

15. Wang H, Huo X, Yang XR, He J, Cheng L, Wang N, Deng X, Jin H, Wang N, Wang C, Zhao F, Fang J, Yao $M$, et al. STAT3-mediated upregulation of IncRNA HOXD-AS1 as a ceRNA facilitates liver cancer metastasis by regulating SOX4. Mol Cancer. 2017;16:136.

16. Lin Y, Jian Z, Jin H, Wei X, Zou X, Guan R, Huang J. Long non-coding RNA DLGAP1-AS1 facilitates tumorigenesis and epithelial-mesenchymal transition in hepatocellular carcinoma via the feedback loop of miR-26a/b-5p/IL-6/JAK2/STAT3 and Wnt/beta-catenin pathway. Cell Death Dis. 2020;11:34.

17. Luo X, Wei J, Yang FL, Pang XX, Shi F, Wei YX, Liao BY, Wang JL. Exosomal IncRNA HNF1A-AS1 affects cisplatin resistance in cervical cancer cells through regulating microRNA-34b/TUFT1 axis. Cancer Cell Int. 2019;19:323.

18. Ding L, Wang L, Li Z, Jiang X, Xu Y, Han N. The positive feedback loop of RHPN1-AS1/miR1299/ETS1 accelerates the deterioration of gastric cancer. Biomed Pharmacother. 2020;124:109848.

19. Wang J, Ding W, Xu Y, Tao E, Mo M, Xu W, Cai X, Chen X, Yuan J, Wu X. Long non-coding RNA RHPN1AS1 promotes tumorigenesis and metastasis of ovarian cancer by acting as a ceRNA against miR596 and upregulating LETM1. Aging. 2020;12:4558-72.

20. Lu L, Yu X, Zhang L, Ding X, Pan H, Wen X, Xu S, Xing Y, Fan J, Ge S, Zhang H, Jia R, Fan X. The Long Non-Coding RNA RHPN1-AS1 Promotes Uveal Melanoma Progression. Int J Mol Sci. 2017; 18.

\section{Figures}

A

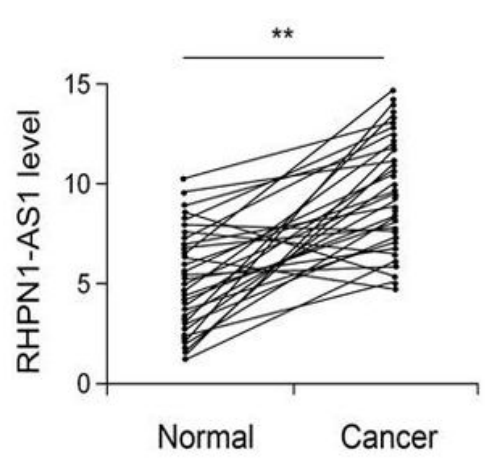

B

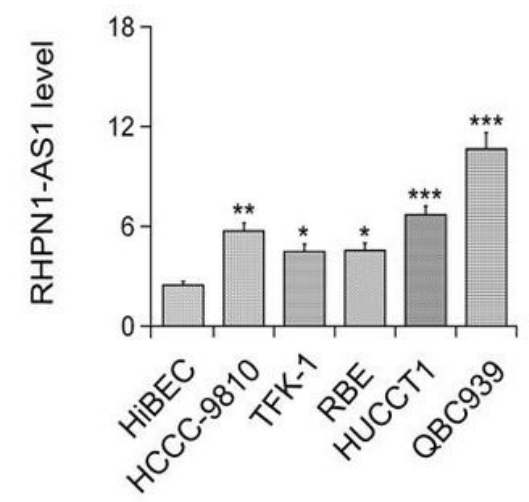

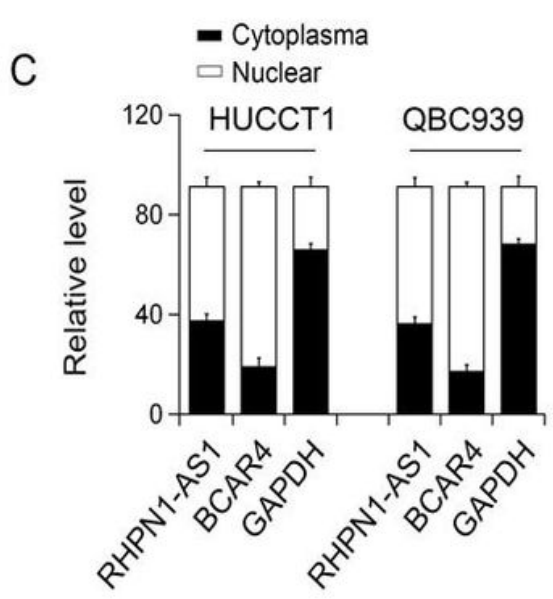


Figure 1

RHPN1-AS1 was upregulated in CCA tissues and cells. A: RHPN1-AS1 expression between CCA samples $(n=34)$ and control samples $(n=34)$. B: RHPN1-AS1 expression in CCA cell lines compared to HiBEC cells determined by qRT-PCR. C: Nuclei location of RHPN1-AS1 expression in CCA cells examined by qRT-PCR.

A
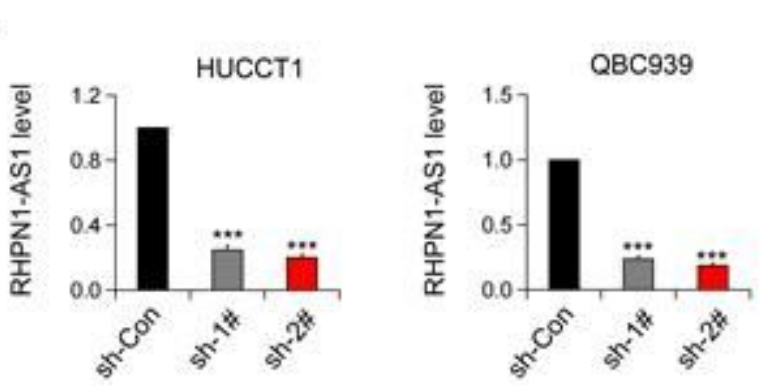

B

HUCCT1

QBC939
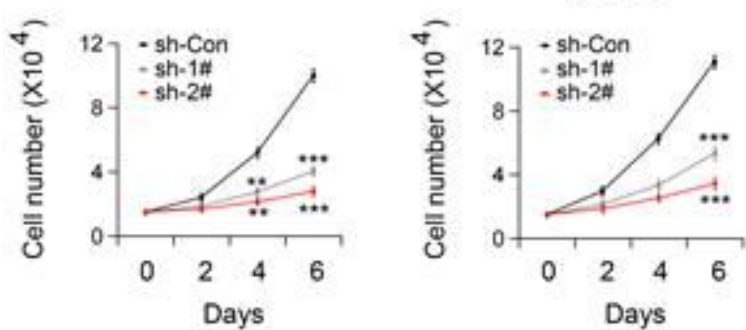

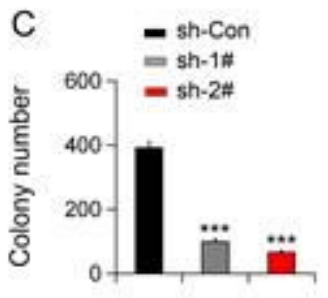

HUCCT1

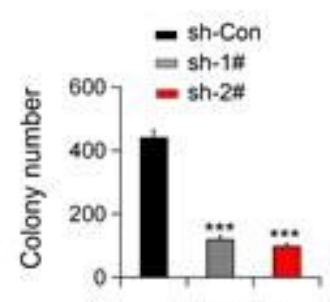

QBC939
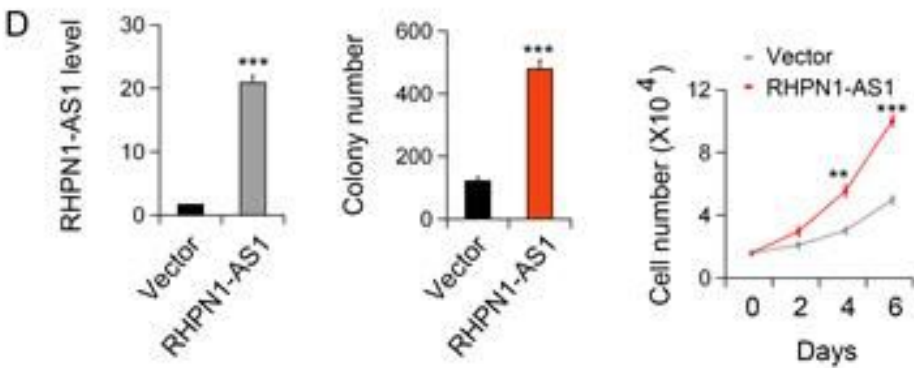

E

sh-Con
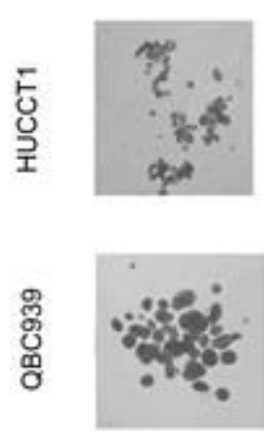

sh-1\#
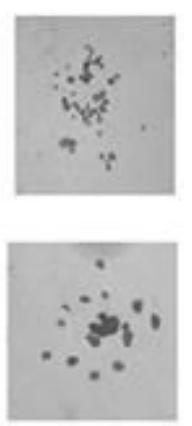

sh-2\#

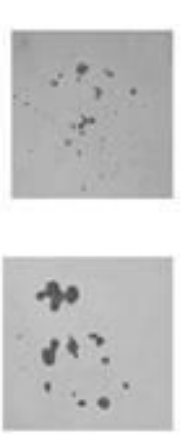

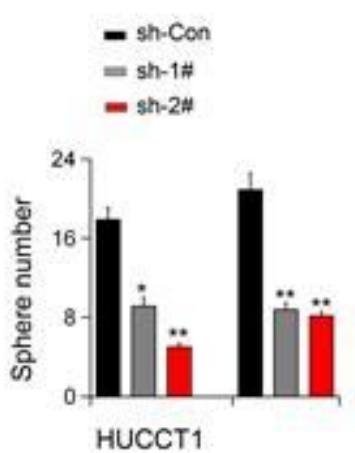

$\mathrm{F}$

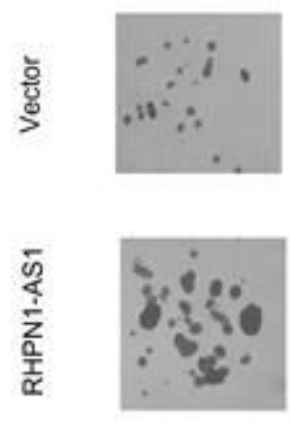

- Vector

- RHPN1-AS1

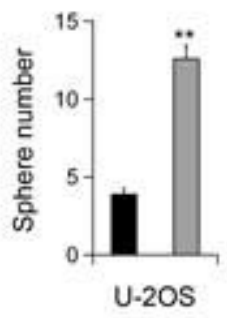

\section{Figure 2}

RHPN1-AS1 promoted CCA cells proliferation and colony formation. A: knockdown of RHPN1-AS1 in CCA cells. B-C: The proliferative and colony-forming capacity were examined in HUCCT1 and QBC939 cells after RHPN1-AS1 knockdown. D: Effect of RHPN1-AS1 overexpression on proliferation and colonyforming capacity in CCA cells. E-F: Effect of RHPN1-AS1 on tumor sphere forming ability of CCA cells. 

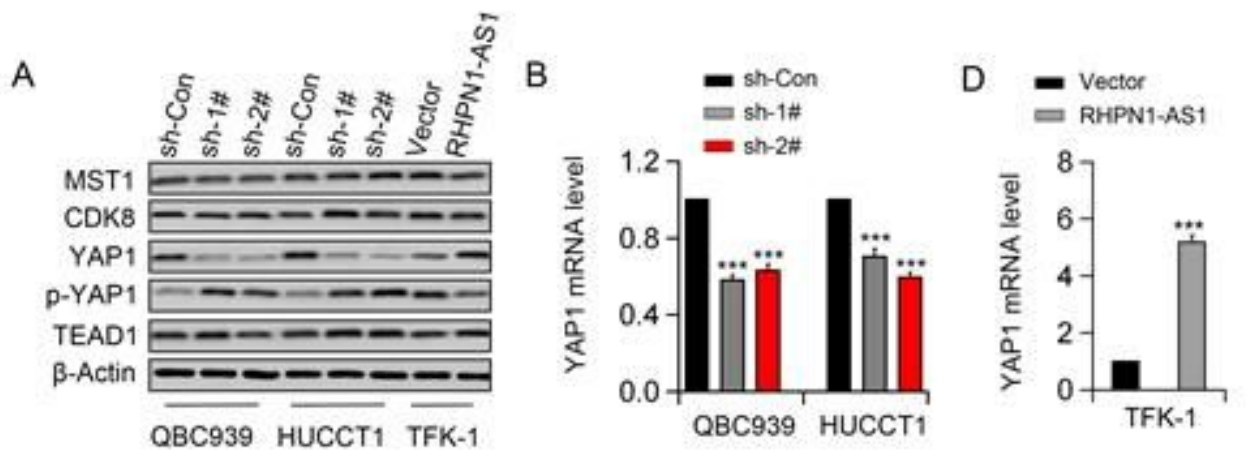

F

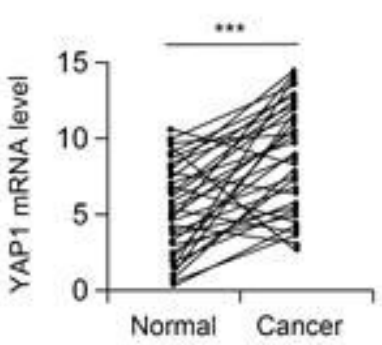

C

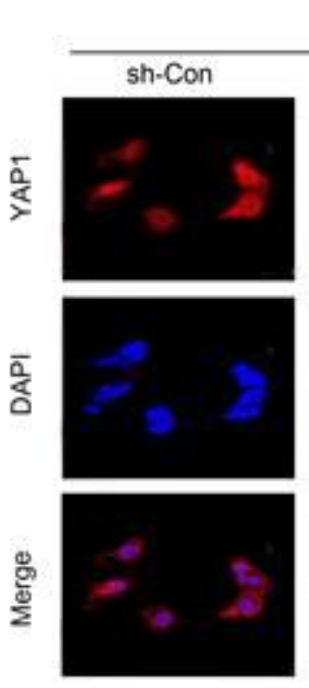

TFK-1
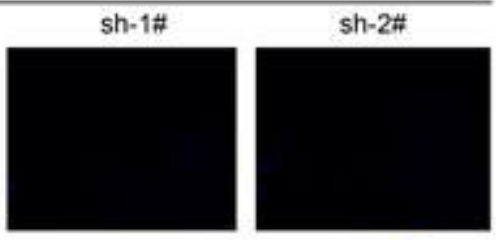

E

TFK-1
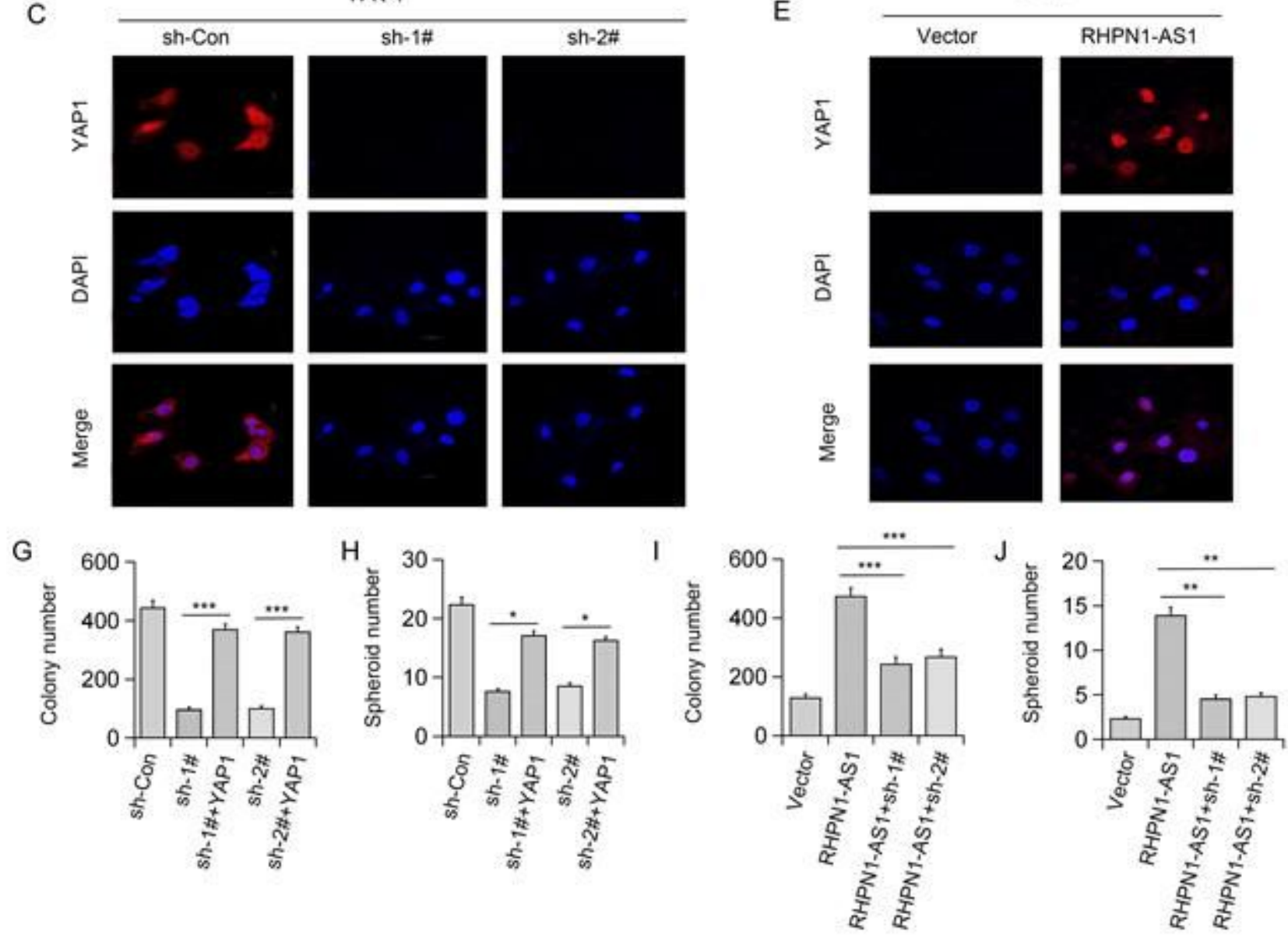

\section{Figure 3}

RHPN1-AS1 overexpression enhanced CCA cell proliferation through YAP1 activation. A-B: The YAP1 mRNA and protein levels were detected by western blot and qRT-PCR after RHPN1-AS1 knockdown. C: Expression of YAP1 after RHPN1-AS1 overexpression in QBC939 and TFK-1 cells detected by Immunofluorescent assay. D: The YAP1 mRNA levels were detected by qRT-PCR after RHPN1-AS1 knockdown in QBC939 and TFK-1 cells. E: Immunofluorescent assay of YAP1 after RHPN1-AS1 overexpression in QBC939 and TFK-1 cells. F: The YAP1 mRNA levels in CCA tissues detected by qRTPCR. G-J: The tumor sphere formation and colony-formation capability of QBC939 and TFK-1 cells. 


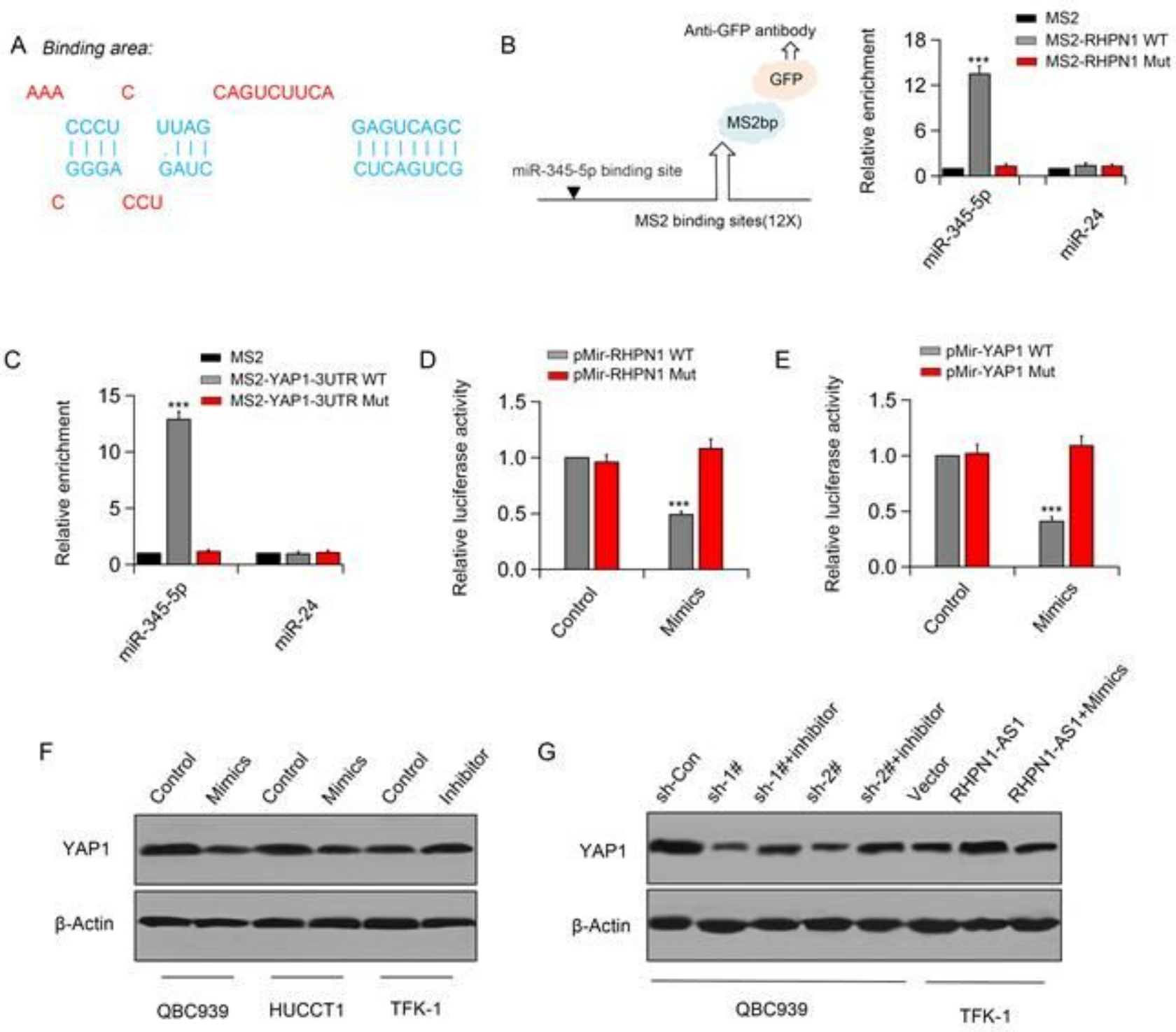

\section{Figure 4}

RHPN1-AS1 upregulated YAP1 expression by sponging miR-345-5p. (A) Targeted sequence between RHPN1-AS1 and miR-345-5p predicted by TargetScan. B: The correlation between miRNAs and RHPN1AS1 detect by MS2-RIP. C: The correlation between miRNAs and YAP1 mRNA 3'-UTR detect by MS2-RIP. DE: Luciferase activities of constructs with RHPN1-AS1-WT or YAP1-WT were reduced by miR-345-5p mimics RHPN1-AS1. F-G: Protein levels of YAP1 were detected by western blot. 
A
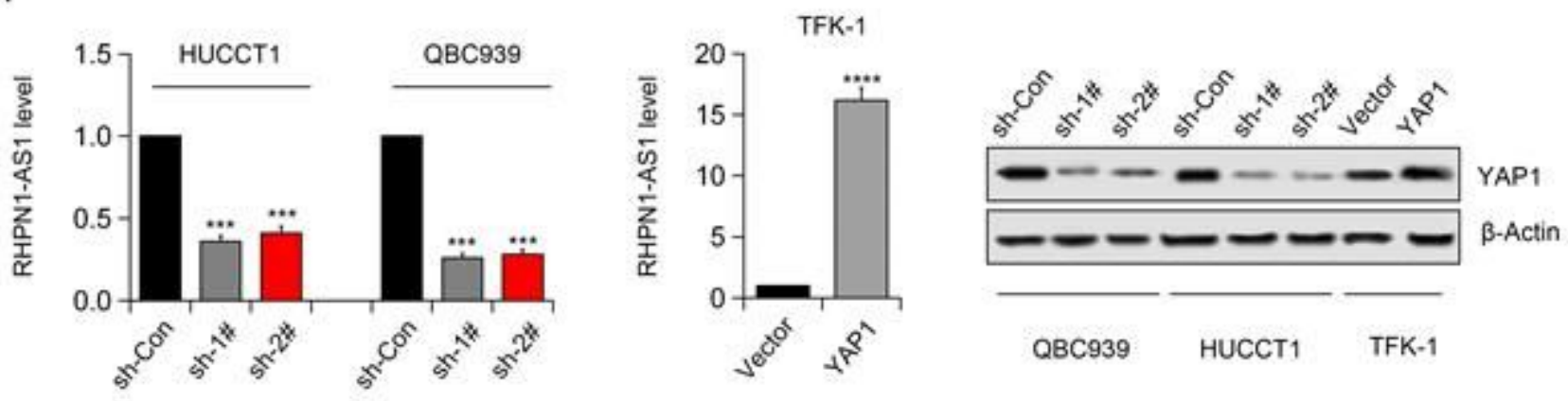

B
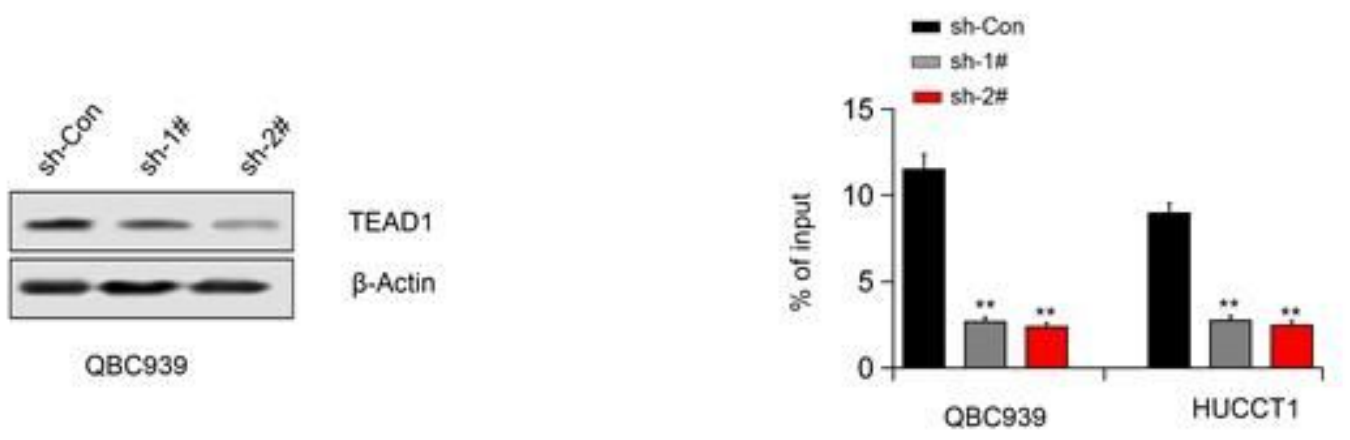

C

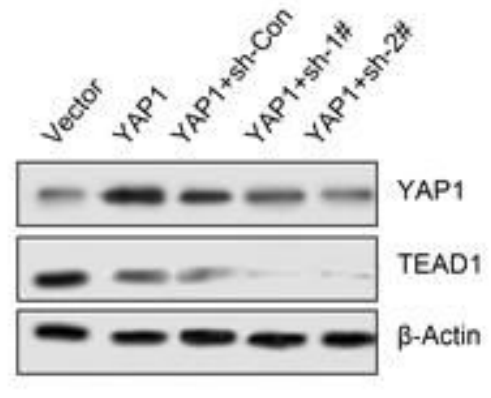

TFK-1

TFK-1

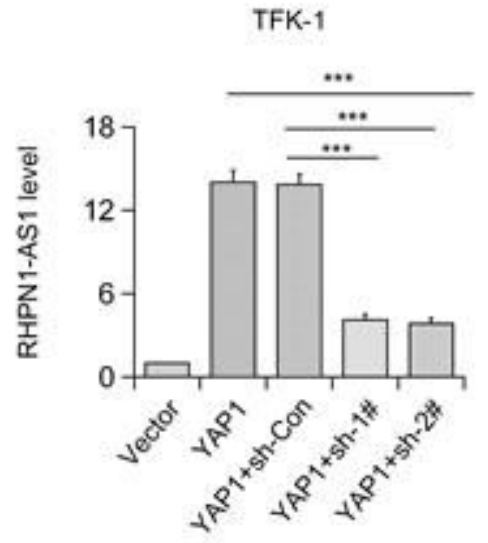

\section{Figure 5}

YAP1/TEAD1 complex feedback transactivated RHPN1-AS1. A: The expression of YAP1 was decreased by RHPN1-AS1 knockdown. B: RHPN1-AS1 level examined by qRT-PCR. C: TEAD1 knockdown reversed RHPN1-AS1 level induced by YAP1 overexpression. ** $p<0.01$, *** $p<0.001$. 


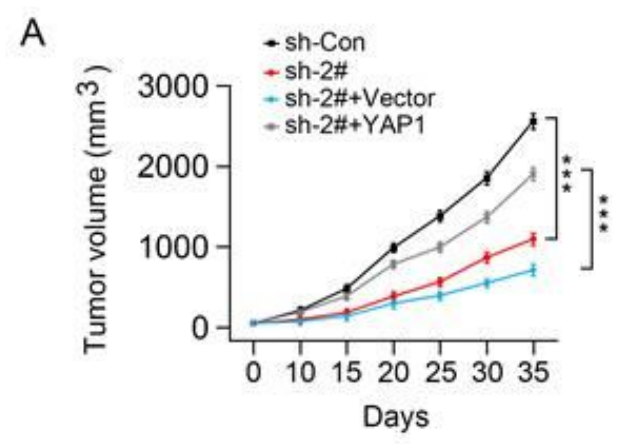

B
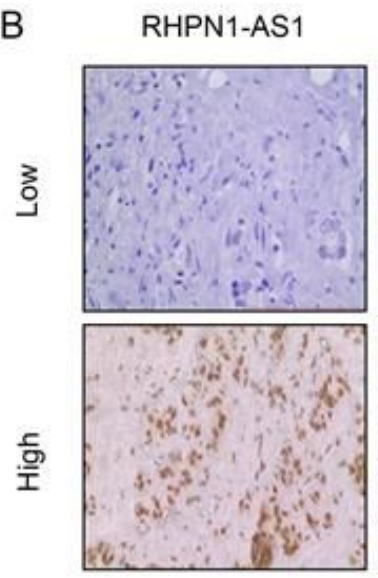

C

\begin{tabular}{ccccc}
\multicolumn{5}{c}{ RHPN1-AS1 } \\
\hline Expression & & High & Low & p value \\
\hline YAP1 & High & 21 & 2 & \\
& Low & 8 & 3 & 0.0084 \\
\hline
\end{tabular}

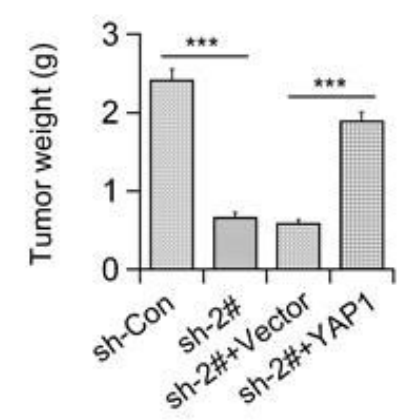

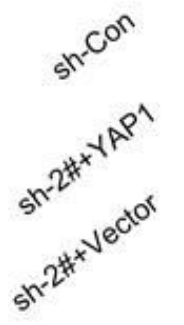

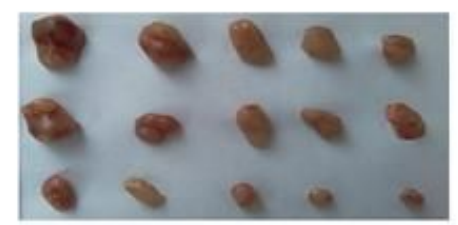

D
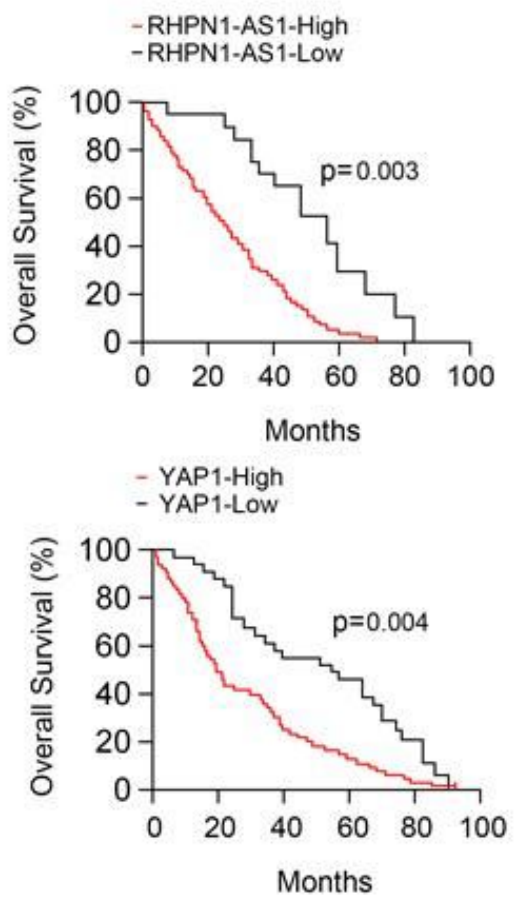

Figure 6

RHPN1-AS1 promoted growth of xenografts in nude mice via YAP1. A: RHPN1-AS1 knockdown suppressed tumor growth in nude mice, whereas rescued by YAP1 expression. B: Expressions of RHPN1AS1 and miR-345-5p in CCA tissues detected by ISH assay. C: Correlation between YAP1 and RHPN1-AS1 in CCA tissues was examined. D: Overall survival of CCA patients detected by Kaplan-Meier analysis. 


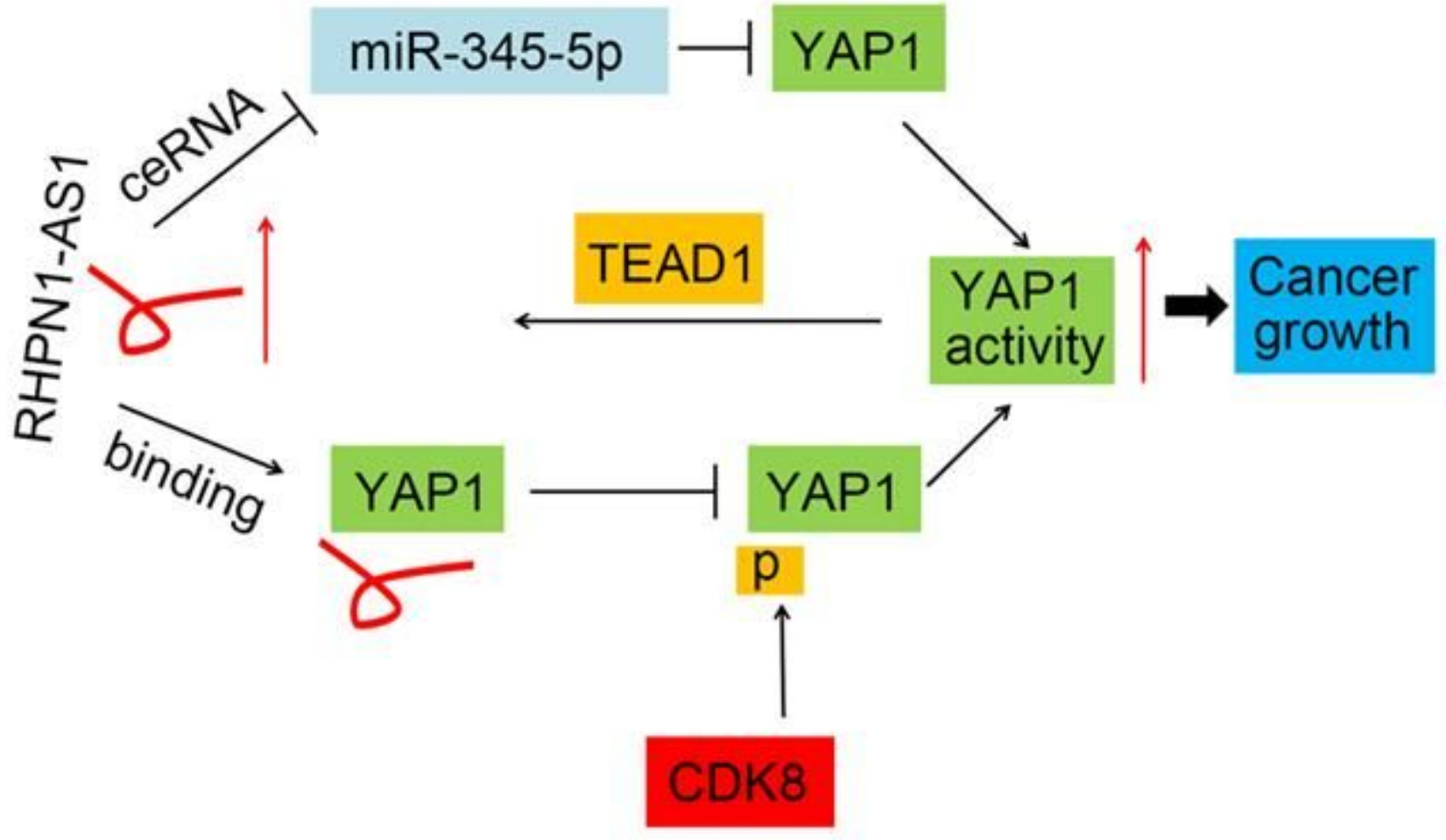

Figure 7

The hypothetical diagram illustrating relationships among RHPN1-AS1/YAP1 signalling pathway. 